\title{
Modelo Determinístico Basado en Indicadores de Condición para Evaluación de Sistemas de Aislación de Bobinados Estatóricos de Generadores Eléctricos
}

\author{
A. E. Barberán ${ }^{\text {a }}$, F. Brítez ${ }^{\text {a }}$, R. G. Aguilar ${ }^{\text {b }}$, M. A. Mazzoletti ${ }^{\text {b }}$, O. Perrone ${ }^{\mathrm{b}}$, M. O. Oliveira ${ }^{\text {b }}$ \\ ${ }^{a}$ Facultad de Ingeniería, Universidad Nacional de Misiones (UNaM), Oberá, Misiones, Argentina. \\ ${ }^{b}$ LIDEE (Laboratorio de Investigación y Desarrollo en Energía Eléctrica) FI-UNaM, Oberá, Misiones, Argentina. \\ * e-mail: lidee@fio.unam.edu.ar
}

\begin{abstract}
Resumen
Este trabajo propone un modelo determinístico para evaluar el estado de condición del Sistema de Aislación del Bobinado Estatórico (SABE) de generadores eléctricos de gran potencia. El modelo propuesto integra diferentes Indicadores de Condición (IC) con el fin de establecer un orden de prioridad de intervención en hidrogeneradores. Con el objetivo de mejorar la planificación de gestión de activos y, de manera particular, programar las tareas de mantenimiento relacionadas con el SABE, un Gestor de Ensayos (GE) para máquinas eléctricas rotativas fue desarrollado en el entorno NI LabView. El GE permite visualizar y comparar los registros históricos de mediciones eléctricas con los límites establecidos por las normas de referencias. Además, posibilita registrar, almacenar y gestionar información de ensayos dieléctricos off-line e inspecciones visuales. A partir del procesamiento de los datos registrados, el GE calcula un único IC para determinar el estado general del SABE. Los resultados obtenidos muestran que el modelo propuesto permite evaluar el estado general del sistema de aislación de los bobinados estatóricos tomando como referencia 8 hidrogeneradores y considerando 14 indicadores de condición.
\end{abstract}

Palabras Clave - Bobinado estatórico, Ensayos dieléctricos, Gestión de activos, Hidrogeneradores, Indicadores de condición, Modelo determinístico, Operaciones de mantenimiento, Sistema de aislación estatórico.

\begin{abstract}
This paper proposes a deterministic model to evaluate the insulation system condition of the stator windings for power electric generators. The proposed model integrates different Condition Indicators in order to establish a priority order of intervention in hydrogenerators. With the aim of improving the planning of asset management and, in particular, the scheduled maintenance tasks related to the stator winding insulation system, a Testing Manager for rotating electrical machines was developed in the environment NI LabView. The Testing Manager allows to visualize and compare the historical records of electric measurements with the limits established by the reference standards. In addition, it makes it possible to manage, record and store off-line dielectric tests data and the visual inspection. From the data processing of the registration electric measurements, the Testing Manager calculates a single condition indicator to determine the general condition of the stator insulation system. The results obtained show that the proposed model allows to evaluate the insulation system state of the with reference to 8 hydrogenerators and considering 14 condition indicators.
\end{abstract}

Keywords - Hydrogenerators, Insulation of the windings, Stator windings, Dielectric tests, Deterministic model, Condition indicators, Asset Management, Maintenance operations.

\section{Introducción}

De acuerdo a un relevamiento internacional realizado en el año 2009 por el CIGRE [1], el Sistema de Aislación del Bobinado Estatórico (SABE) de un hidrogenerador presenta un mayor porcentaje de fallas en comparación con otros componentes de la máquina. En [1] se analizaron

\footnotetext{
*Autor en correspondencia.
} 
cerca de 1200 hidrogeneradores instalados en 5 países revelando que, entre todas las posibles causas de falla en una máquina de este tipo, el $56 \%$ de las fallas se originan en el sistema de aislación estatórico y el restante se clasifican en fallas de origen mecánico (24\%), térmico (17\%) y en cojinetes $(3 \%)$.

En este sentido, en los últimos años se han desarrollado alrededor de 40 ensayos y verificaciones específicas para el control del sistema de aislación estatórico [2]. Una de las metodologías más difundidas que emplea Indicadores de Condición (IC) para la gestión del mantenimiento de activos en centrales hidroeléctricas corresponde al EPRI (Electric Power Research Institute) la cual se expone en la guía Hydro Life Extension Modernization Guide - Volume 3 Electromechanical Equipment - Appendix D [3]. Esta metodología utiliza una serie de ensayos e inspecciones altamente invasivas que requieren el desmontaje de gran parte del generador para realizar el diagnostico, lo cual no siempre es posible dado el costo asociado y las dificultades técnicas que implica una intervención mayor en este tipo de máquinas.

En particular, en las centrales de generación, el departamento de gestión de activos se vale de ensayos y verificaciones del tipo on-line/off-line para determinar el grado de avance de los mecanismos de degradación que afectan la integridad dieléctrica de los materiales aislantes empleados en el bobinado estatórico. Por lo tanto, el trabajo propuesto se focaliza en el estudio de los IC que se utilizan para inferir en el estado operacional del SABE de generadores síncronos de gran porte refrigerados en aire. En este sentido, a partir de la información disponible de una serie de ensayos, verificaciones e inspecciones visuales fue posible evaluar y determinar el estado de la condición del sistema de aislación estatórico de 8 hidrogeneradores. Para ello, se asignó una escala de puntajes de acuerdo a una escala de valoración definida para cada ensayo y al mismo tiempo brindar un orden de prioridad de intervención para la elaboración de un cronograma de ejecución de operaciones de mantenimiento.

\section{Definición de Indicadores de Condición (IC) Considerados}

La determinación de un orden de prioridad de intervención para operaciones de mantenimiento en máquinas rotantes, requiere el estudio en conjunto de una multiplicidad de variables indicadoras de la magnitud y grado de evolución de los mecanismos de degradación que se encuentran activos. De manera particular, el diagnóstico de la condición del sistema de aislación estatórico, por la compleja interrelación entre los mecanismos de degradación actuantes, será más preciso cuanto mayor sea el número de variables analizadas.

Teniendo en cuenta las dificultades técnico-económicas de aplicación de la metodología EPRI, mencionada anteriormente, la metodología que se presenta en este trabajo considera 14 indicadores basados en ensayos e inspecciones que requieren un mínimo de intervención. En la Tabla 1 se presentan los IC (ensayos/inspecciones) empleados por el EPRI y los utilizados por la metodología propuesta. 
Tabla 1. IC utilizados en la metodología propuesta e IC utilizados por EPRI

\begin{tabular}{l|l}
\hline \multicolumn{1}{c|}{ Metodología Propuesta (14 IC) } & \multicolumn{1}{c}{ EPRI (11 IC) } \\
\hline Resistencia de Aislación, Indice de polarización y de absorción, & Blackout Test, Corona Probe Test, DC Hi-Pot Test, \\
Pendiente final SV, Perfil de polarización SV, & Insulation Resistance, Ozone Detection Test, \\
Tangente delta Tip-Up ( $\Delta \mathrm{TD})$, Capacidad Tip-Up $(\Delta \mathrm{C})$, & $\begin{array}{l}\text { Partial Discharge Test, Circuit Ring Inspection, } \\
\text { Core Inspection, End turn Inspection, Lead Inspection, } \\
\text { Descargas parciales (DP), Energía de las DP, Clase de DP (CDP) } \\
\text { Tensión de encendido de las DP, Concentración de O3, }\end{array}$ \\
Frecuencia de paradas (FP), $\Delta \mathrm{T}$ de bobinados. & Wedge System Inspection. \\
\hline
\end{tabular}

La selección de los IC evaluados en este trabajo, ha sido realizada considerando los cuatro mecanismos de degradación actuantes sobre el SABE de las máquinas eléctricas rotantes refrigeradas en aire, a saber: Térmico, Eléctrico, Ambiental y Mecánico (TEAM), los cuales se describen a seguir. Las Tablas siguientes presentan la valoración de los IC junto a una escala de colores que orienta sobre el estado operacional (verde: muy bueno, amarillo: bueno, naranja: regular, rojo: deficiente).

\subsection{Degradación Térmica}

La sobreelevación de temperatura que experimenta el bobinado estatórico durante el funcionamiento de las máquinas eléctricas, activa reacciones químicas de oxidación en el material aislante. Esto deriva en procesos de delaminación, es decir, en la separación de las diferentes capas de material aislante por pérdida de la rigidez de unión del material de impregnación.

Los IC sensibles a la presencia de mecanismos de degradación térmica son los siguientes:

- $\quad \Delta$ TD, $\Delta \mathbf{C}$ : representan los valores tip-up de tangente delta y capacidad entre el $60 \%$ y el $20 \%$ de la tensión nominal de línea. La Tabla 2 presenta la valoración propuesta para estos indicadores.

Tabla 2. Valoración de los Indicadores $\Delta \mathrm{TD}$ y $\Delta \mathrm{C}$

\begin{tabular}{|c|c|c|c|}
\hline \multicolumn{2}{|c|}{$\Delta \tan \delta(\Delta T D)$} & \multicolumn{2}{|c|}{$\Delta C$ apacidad $(\Delta C)$} \\
\hline Niveles [\%] & Valoración & Niveles $[\%]$ & Valoración \\
\hline$A T D=03 \%$ & 1 & If $\quad 0,3 \%$ & 1 \\
\hline $0.3^{\circ} / \mathrm{a}=\Delta T \mathrm{D}=0.5 \%$ & 3 & $0.3^{\prime \prime} 0^{\circ}=\mathrm{MC}<1.9 .5^{\prime \prime}$ & 2 \\
\hline $0,5 \%<\Delta \mathrm{TD}<0,8 \%$ & 3 & $0,5 \%<\Delta C<0,8 \%$ & 3 \\
\hline $0,8 \%<\Delta \mathrm{TD}<1 \%$ & 4 & $0.8 \%<\Delta C<1 \%$ & 4 \\
\hline AID & 5 & $\Delta \mathrm{C}-1^{\mathrm{a}}$ & 5 \\
\hline$\Delta / D \leq 1 / 4 y$ & 5 & AC $50^{\circ}$ & 5 \\
\hline
\end{tabular}

- $\Delta \mathbf{T}$ : Temperatura máxima registrada en el bobinado. Se valora con respecto al nivel admisible según la clase de aislación de la máquina. La Tabla 3 presenta la valoración propuesta para estos indicadores.

Tabla 3. Valoración del indicador $\Delta \mathrm{T}$

\begin{tabular}{c|c}
\hline \multicolumn{2}{c}{ Elevación de Temperatura del Bobinado Estatórico $(\Delta \mathbf{T})$} \\
\hline Niveles $\left[{ }^{\circ} \mathrm{C}\right]$ & Valoración \\
\hline$\Delta \mathrm{T}<85$ & 1 \\
$85<\Delta \mathrm{T}<100$ & 2 \\
\hline $100<\Delta \mathrm{T}<130$ & 3 \\
\hline $\mathrm{T} \quad 130$ & 5 \\
\hline
\end{tabular}




\subsection{Degradación Eléctrica}

El proceso de degradación eléctrica se origina por la actividad de Descargas Parciales (DP) las cuales se producen en oclusiones gaseosas dentro del material aislante como consecuencia de imperfecciones en el proceso de fabricación de las bobinas. Específicamente, la degradación se produce por el bombardeo de iones y electrones que rompen progresivamente las uniones químicas del material aislante, derivando en una ruptura dieléctrica del sistema de aislación.

Los IC sensibles a la presencia de mecanismos de degradación eléctrica son los siguientes:

- EDP: Energía integrada de las descargas al $60 \%$ de la tensión nominal de línea de la máquina. Según procedimiento ASTM D 3382 [4]. La Tabla 4 presenta la valoración propuesta para este indicador.

Tabla 4. Valoración del indicador EDP

\begin{tabular}{c|c}
\hline \multicolumn{2}{c}{ Energía de las DP (EDP) } \\
\hline Niveles $(\mu \mathrm{J} / \mathrm{pF})$ & Valoración \\
\hline $\mathrm{EDP}-0,5$ & 1 \\
\hline $0,5 \quad \mathrm{DP} \quad 1,5$ & 2 \\
\hline $1,5<\mathrm{EDP}<3,5$ & 3 \\
\hline $3,5<\mathrm{EDP}<6$ & 4 \\
\hline $\mathrm{DP}<0$ & 5 \\
\hline
\end{tabular}

- DPp y CDP: Representan la magnitud y tipo de DP detectadas. Se evalúan en función del límite práctico de 10 a $12 \mathrm{nC}$ al $60 \%$ de la tensión nominal de línea de la unidad para un funcionamiento confiable y de acuerdo con la IEC 60034-27 [5] la cual establece niveles de riesgo para cada tipo de descarga que pueda presentarse. La Tabla 5 presenta la valoración propuesta para estos indicadores.

- UE: o tensión de encendido de las DP, es la tensión de inicio de actividad de DP. La Tabla 6 presenta la valoración propuesta para este indicador.

Tabla 5. Valoración de indicadores DPp y CDP.

\begin{tabular}{|c|c|c|c|}
\hline \multicolumn{2}{|c|}{ Descargas parciales permanentes (DPp) } & \multicolumn{2}{|c|}{ Clase de Descargas parciales(CDP) } \\
\hline Niveles $[\%]$ & Valoración & Niveles & Valoración \\
\hline$D P=+$ & 1 & lintertiks (I) & 1 \\
\hline $4<\mathrm{DP}<8$ & 2 & $\begin{array}{c}\text { I+ Corona (Cabezal - entre } \\
\text { barras) }\end{array}$ & 3 \\
\hline $8<\mathrm{DP}<12$ & 3 & I+ Delaminación interna & 3 \\
\hline $12<\mathrm{DP}<20$ & 4 & $\begin{array}{l}\text { 1-Delaminacion conductor: } \\
\text { - utslante }\end{array}$ & 5 \\
\hline$D P=30$ & 5 & fincrnas Kamurn $(\mathrm{R})$ & 5 \\
\hline
\end{tabular}

Tabla 6. Valoración de indicador UE.

\begin{tabular}{c|c}
\hline \multicolumn{2}{|c|}{ Tensión de encendido (UE) } \\
\hline Niveles [V] & Valoración \\
\hline UEC & 1 \\
\hline $3000<$ UEC $<3500$ & 2 \\
\hline $2800<$ UEC $<3000$ & 3 \\
\hline$E C<2804$ & 5 \\
\hline
\end{tabular}




\subsection{Degradación Ambiental}

La degradación por contaminación con polvo ambiente, residuos de carbón de escobillas y humedad que se depositan sobre la superficie del sistema de aislación, no provocan por sí mismas la degradación del material aislante de las bobinas. Sin embargo, facilitan la degradación por otros mecanismos, por ejemplo, degradación eléctrica manifestada como tracking superficial (descargas parciales superficiales) debido a contaminación. Dentro de los mecanismos de degradación ambiental se encuentra la degradación química originada por la producción de ozono como consecuencia de las DP superficiales que pueden manifestarse como: DP en ranura, DP en la zona de solape de película semiconductora con pintura graduadora de potencial, DP entre barras adyacentes y como tracking superficial en cabezas de bobina [6]. El ozono en combinación con la humedad ambiente y el nitrógeno del aire produce ácido nítrico (HNO3) que luego ataca químicamente a los materiales epoxídicos que componen el material aislante.

De esta manera, los IC sensibles a la presencia de degradación ambiental son:

- RA, IP, IA: estos índices se valoraron de acuerdo a los criterios de IEEE Std. 43 - 2013. La Tabla 7 presenta la valoración propuesta para estos indicadores.

- ST, PST: representan las características de la curva de polarización del ensayo de saltos de tensión, evaluándose la pendiente de crecimiento (ST) y la tendencia o perfil de la curva (PST). La Tabla 8 presenta la valoración propuesta para estos indicadores.

Tabla 7. Valoración de indicadores RA, IP e IA.

\begin{tabular}{|c|c|c|c|c|c|}
\hline \multicolumn{2}{|c|}{ Resistencia de Aislación (RA) } & \multicolumn{2}{|c|}{ İndice de polarización (IP) } & \multicolumn{2}{|c|}{ Indice de absorción (IA) } \\
\hline Niveles $[\mathrm{M} \Omega]$ & Valoración & Niveles [M $\Omega]$ & Valoración & Niveles [M $\Omega]$ & Valoración \\
\hline $\mathrm{RA}=1000$ & 1 & II $=4$ & 1 & $1 \mathrm{~A}=1.6$ & 1 \\
\hline $600<\mathrm{RA}<1000$ & 2 & $3<1 \mathrm{IP}-4$ & 3 & $1.4<1 \mathrm{1}<1.6$ & 2 \\
\hline $300<\mathrm{RA}<600$ & 3 & $2<\mathrm{IP}<3$ & 3 & $1,25<\mathrm{IA}<1,4$ & 3 \\
\hline $100<\mathrm{RA}<300$ & 4 & $1,5<\mathrm{IP}<2$ & 4 & $1,1<\mathrm{IA}<1,25$ & 4 \\
\hline RA $\leq 100$ & 5 & IP $\leq 1,3$ & 5 & $1 \mathrm{~A}-1.1$ & 5. \\
\hline
\end{tabular}

Tabla 8. Valoración de indicadores ST y PST

\begin{tabular}{|c|c|}
\hline \multicolumn{2}{|c|}{ Saltos de tensión (ST) } \\
\hline Niveles $[\%]$ & Valoración \\
\hline Pendicnte $>1000^{\circ}$ & 1 \\
\hline $80^{\circ}$ in $>$ Pendionte $>1000^{\circ}$ & 2 \\
\hline $60 \%>$ Pendiente $>80 \%$ & 3 \\
\hline $40 \%<$ Pendiente $<60 \%$ & 4 \\
\hline Pendicute $40 \%$, & 5 \\
\hline \multicolumn{2}{|c|}{ Perfil Saltos de tensión (PST) } \\
\hline Niveles & Valoración \\
\hline Constantemente creviente & 1 \\
\hline Creciente inestable & 2 \\
\hline Decrectisate constinte & 3 \\
\hline
\end{tabular}

- Concentración de O3: la concentración de ozono ha sido dividida en tres valoraciones según valores propuestos por EPRI. Baja: concentraciones menores a 0,1 ppm. Moderado: concentraciones entre 0,1 y $0,6 \mathrm{ppm}$. Alto: concentraciones entre 0,6 y $0,8 \mathrm{ppm}$. 
Concentraciones mayores a $0,8 \mathrm{ppm}$ son consideradas excesivas y requieren ventilación forzada por cuestiones de seguridad. La Tabla 9 presenta la valoración propuesta para estos indicadores.

Tabla 9. Valoración de indicador 03

\begin{tabular}{c|c}
\hline \multicolumn{2}{|c|}{ Concentración de Ozono $\mathrm{O}_{3}$} \\
\hline Niveles & Valoración \\
\hline Bayo $(0.1 \mathrm{ppm})$ & 1 \\
\hline Moderado $\left(0,1<\mathrm{O}_{3}<0,6 \mathrm{ppm}\right)$ & 2 \\
\hline Alto $(0,6(0,-0,8 \mathrm{pm})$ & 3 \\
\hline
\end{tabular}

\subsection{Degradación Mecánica}

Considerando el sistema de aislación estatórico podemos distinguir fundamentalmente dos fuentes de estrés mecánico. La primera es originada por la interacción electromagnética entre el rotor y el estator a través del flujo magnético de entrehierro y las fuerzas de atracción y repulsión experimentada en el bobinado, especialmente en la zona de cabezas de bobina la cual pulsa a una frecuencia igual al doble de la frecuencia de generación. Este mecanismo de degradación es de acción permanente durante la operación de la unidad. En segundo lugar, se encuentra la degradación mecánica originada en las maniobras de arranque/parada y rechazos de carga de la unidad las cuales son de actuación temporal. En estas operaciones pueden presentarse transitorios de corriente superiores a los valores nominales lo que se traduce en fuerzas de origen electromagnético que superan ampliamente las existentes en operación normal [2].

Estas fuentes de estrés mecánico afectan la integridad del material aislante de las bobinas por abrasión, debido a las vibraciones de las bobinas en las ranuras y al agrietamiento del aislante producto de la deflexión de las cabezas de bobina, causadas por las fuerzas de atracción/repulsión.

De las dos fuentes de degradación mecánica, se han considerado solamente las del segundo grupo debido a la indisponibilidad de datos de vibraciones del núcleo estatórico y/o cabezas de bobina para el caso presentado. De esta manera el IC sensible a la degradación mecánica es función del número de ciclos de arranque/parada por año:

Frecuencia de arranque/parada (FP): Valora el promedio anual de arranques y paradas de una maquina en particular con respecto al nivel recomendado por el fabricante, el cual recomienda una parada cada 35.000 hs de servicio para el caso de estudio presentado. La Tabla 10 presenta la valoración propuesta para este indicador.

Tabla 10. Valoración de indicador FP

\begin{tabular}{c|c|}
\hline \multicolumn{2}{|c}{ Frecuencia de paradas } \\
(FP - promedio paradas por año) \\
\hline Niveles & Valoración \\
\hline $\mathrm{FP}<0,25$ & 1 \\
\hline $0,25<\mathrm{FP}<2$ & 2 \\
\hline $2<\mathrm{FP}<3,5$ & 3 \\
\hline $3,5<\mathrm{FP}<5$ & 4 \\
\hline $\mathrm{PP}<5$ & 5 \\
\hline
\end{tabular}




\section{Modelo Propuesto para Integración de Indicadores de Condición}

Los indicadores presentados desde la Tabla 2 a la Tabla 10 se integran por medio de un modelo determinístico con el objetivo de establecer un Orden de Prioridad (OP) de intervención por comparación entre las máquinas analizadas. Si bien cada indicador considerado presenta una misma escala de valoración, en el proceso de integración los mismos han sido ponderados en función de su relación con los fenómenos de degradación que pudieran estar desarrollándose en las máquinas. En este estudio cada indicador se agrupó en términos correspondientes al tipo de ensayo del cual deriva. El grado de importancia se pondero por medio de un coeficiente entre 0 y 1 , cuyo valor fue consensuado, de acuerdo a la información que brinda y a la importancia relativa de cada ensayo en el diagnóstico de condición de la máquina.

El modelo propuesto para la determinación del OP de las máquinas se obtuvo por medio de la agrupación de los distintos ensayos realizados (de corriente continua y corriente alterna) y la información operativa disponible de la maquina (arranques/paradas y temperatura máxima registrada). De esta forma, el modelo determinístico propuesto para la integración de los IC que resulten en la definición de un Orden de Prioridad (OP) se expresa a través de la siguiente ecuación:

$$
O P=a(W)+b(X)+c(Y)+d(Z)
$$

donde:

$$
\begin{aligned}
& W=F P+\mathrm{D} T \\
& X=R A+I P+I A+S T+P S T \\
& Y=\mathrm{D} T D+\mathrm{DC} \\
& Z=D P+C D P+U E C+E D P+O_{3} \\
& a=0,35 ; b=0,1 ; c=0,2 ; d=0,35
\end{aligned}
$$

El $1^{\circ}$ término de la ecuación (1) corresponde a la información operativa disponible de cada unidad, representativo de mecanismos de degradación mecánica. El $2^{\circ}$ término agrupa los indicadores de los ensayos de corriente continua representativos del grado de contaminación del sistema de aislación estatórico. El $3^{\circ}$ término agrupa los indicadores del ensayo de tangente delta y capacidad los cuales son sensibles a la presencia de mecanismos de degradación térmica. El $4^{\circ}$ término agrupa los índices relacionados con el ensayo de descargas parciales, cuya presencia en principio es una manifestación directa de imperfecciones en el SABE.

Cada uno de los términos de la ecuación (1) es un parámetro representativo del daño sobre el sistema de aislación estatórico debido a factores de origen térmico, eléctrico, ambiental y mecánico. Los cuatro términos se obtienen mediante la sumatoria de los pesos individuales de cada indicador correspondiente a su grupo, para luego ser multiplicados por un coeficiente $(a, b, c$ y $d)$ valorados entre 0 y 1 , que pondera la importancia relativa respecto a los demás términos que intervienen en la ecuación. En este trabajo los coeficientes propuestos y asignados a cada coeficiente se obtuvieron de numerosas experiencias realizadas por expertos en intervención de máquinas, de consultas a especialistas en el diagnóstico de máquinas eléctricas rotativas y de guías de referencias internacionales. 


\section{Aplicación de la metodología propuesta: Estudio de Caso}

\subsection{Orden de prioridad para la planificación de la gestión de activos}

La metodología propuesta fue aplicada sobre 8 hidrogeneradores de características similares con el objetivo de determinar un orden de prioridad de intervención para la planificación de un cronograma de mantenimiento programado (overhaul). Los ensayos e inspecciones fueron realizados en cada generador empleando un total de 18 horas de parada por unidad. La información obtenida de dichos ensayos fue valorada según las escalas propuestas en las Tabla 2 a la Tabla 10. En la Tabla 11 se resumen los resultados obtenidos y en la Fig. 1 se muestra el orden de prioridad para la intervención de mantenimiento.

Tabla 11. Matriz de Valoración de Indicadores de Condición

\begin{tabular}{|c|c|c|c|c|c|c|c|c|}
\hline \multicolumn{9}{|c|}{ Orden de Prioridad (OP) para las Maquinas Analizadas } \\
\hline \multirow{2}{*}{ IC } & \multicolumn{8}{|c|}{ Generadores } \\
\hline & $\# 1$ & $\# 2$ & $\# 3$ & $\# 4$ & $\# 5$ & $\# 6$ & $\# 7$ & $\# 8$ \\
\hline RA & 1 & 1 & 2 & 2 & I & 1 & 2 & 2 \\
\hline IP & 1 & 1 & 1 & 1 & 1 & 1 & 3 & 1 \\
\hline IA & 1 & 1 & 1 & 2 & 1 & I & 3 & 1 \\
\hline ST & 4 & 1 & 3 & 2 & 1 & 1 & 5 & 3 \\
\hline PST & 1 & 1 & 1 & 1 & 1 & 1 & 2 & 1 \\
\hline$\Delta \mathrm{TD}$ & 2 & 3 & 1 & 5 & 3 & 2 & 5. & 1 \\
\hline$\Delta C$ & 2 & 3 & 1 & 5 & 4 & 2 & $z$ & 1 \\
\hline DP & 5 & 3 & 2 & 2 & 3 & 2 & 2 & 2 \\
\hline CDP & 5. & 5 & 1 & 5 & 5 & 1 & 3 & 1 \\
\hline EDP & 3 & 2 & I & 1 & 3 & 2 & 3 & $d$ \\
\hline O3 & 3 & 3 & 0 & 3 & 5 & 11 & 0. & 0 \\
\hline UEC & 3 & 3 & 1 & 2 & 2 & 2 & 1 & 1 \\
\hline FP & 4 & 3 & 3 & 3 & 3 & 3 & 2 & 3 \\
\hline$\Delta \mathrm{T}$ & 2 & 1 & 2 & 2 & 2 & 2 & 2 & 2 \\
\hline OP & 10,3 & 8,7 & 4,7 & 9,1 & 9,9 & 5,5 & 7,9 & 4,7 \\
\hline
\end{tabular}

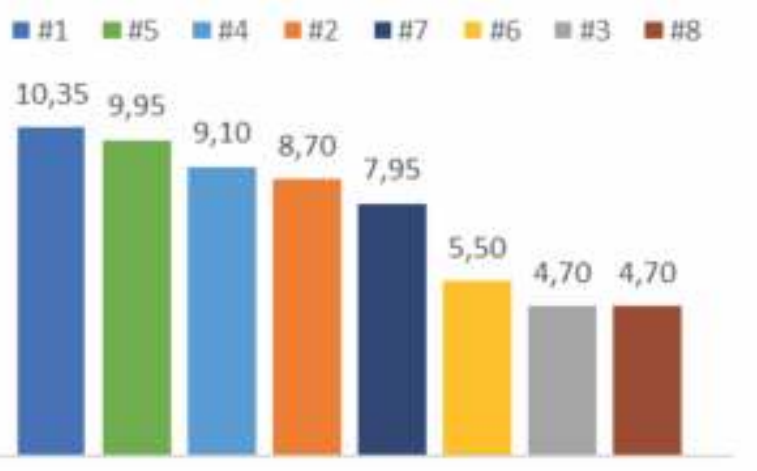

Orden de Prioridad de Intervención

Fig. 1. Orden de prioridad para intervención por mantenimiento. 
Los resultados revelan que las unidades $\# 1, \# 2$, \#4 y \#5 requieren una pronta intervención dado que presentan elevada concentración de ozono en el recinto del generador como consecuencia de la actividad de DP superficiales que predominan en la zona de ranuras. Estas DP pueden deberse a barras flojas por acuñado deficiente, deficiencia en la calidad de la película semiconductora y/o contacto pobre entre el recubrimiento externo de las barras y el núcleo estatórico [7]. Dado que este tipo de degradación es de difícil mitigación [8], la norma IEC 60034-27 clasifica a este tipo de descarga como de "riesgo elevado", por lo cual se le asignó la puntuación máxima al índice CDP (Clase de Descargas Parciales). Por su parte, la unidad \#1 presenta además magnitudes de DP que superan ampliamente el límite recomendado por la práctica. El origen de la actividad de DP en ranura por pérdida de película semiconductora, puede deberse a una deficiencia en la calidad del material semiconductor y/o vibraciones de las bobinas debido a cuñas de cierre de ranura flojas, que provocan erosión mecánica de la superficie de las barras Roebel. Por otro lado, la unidad \#4 ha manifestado valores decrecientes de $\tan \delta$ con el incremento de tensión. Este tipo de comportamiento es característico de un sistema de aislación degradado, probablemente con múltiples cavidades gaseosas y contaminación debido a humedad.

Entre las acciones de mantenimiento recomendadas para las primeras cuatro unidades que lideran el orden de prioridad, se encuentran: revisión mayor del sistema de aislación estatórico, esto incluye una detallada inspección visual del sistema de aislación en la zona cabezas de bobina y salidas de ranura en búsqueda de actividad de DP por efecto corona, mapeo de cuñas, revisión de la integridad de película semiconductora y suplementos en ranura mediante inspección boroscópica.

Siguiendo con el orden de prioridad determinado mediante el modelo propuesto, se encuentra en $5^{\circ}$ lugar la unidad \#7. En este generador se han encontrado indicios de contaminación superficial manifestado por una curva de saltos de tensión (ST) decreciente, lo cual se ve reflejado en los indicadores de Índice de Polarización (IP), Resistencia de Aislación (RA) y Perfil de Saltos de Tensión (PST). Por otro lado, la misma unidad ha manifestado valores de $\Delta \tan \delta$ y $\Delta \mathrm{C}$ superiores al límite recomendado por la práctica [2], indicativo de procesos de degradación térmica. Así mismo, en una de sus fases, la unidad en cuestión ha registrado patrones de DP característico de actividad de descargas en la salida de ranuras, en la zona de solape de película semiconductora con película graduadora de potencial.

Las acciones de mantenimiento que la práctica recomienda en el caso de la unidad \#7 son: inspección visual detallada haciendo especial énfasis en la zona de salidas de ranura, cruce de bobinas y puentes de conexión. De ser necesario, reponer la pintura graduadora de potencial a la salida de ranura. Posteriormente continuar con la limpieza de cabezas de bobina empleando solventes dieléctricos y/o hielo seco (Dry Ice Blasting) según el grado de contaminación que presente el bobinado.

Finalmente, las restantes tres unidades que componen el conjunto de generadores analizados, presentan parámetros de degradación normales para los años de funcionamiento, diferenciándose la unidad \#6 de la \#3 y \#8 únicamente en el índice que valora la variación de tan $\delta$ y Capacidad. Para estas unidades se recomienda realizar las actividades de mantenimiento de acuerdo con las especificaciones del fabricante para las horas de funcionamiento correspondientes. 


\subsection{Desarrollo de un gestor de ensayos dieléctricos para máquinas eléctricas rotativas}

El gestor de ensayos dieléctricos para máquinas eléctricas rotativas (GE) desarrollado en el entorno NI LabView se muestra en la Fig. 2. La ventana principal cuenta con la barra de tareas personalizada que posibilita la carga de datos especificando todos los ensayos correspondientes a la máquina, ya sea de una nueva medición o de mediciones anteriores.

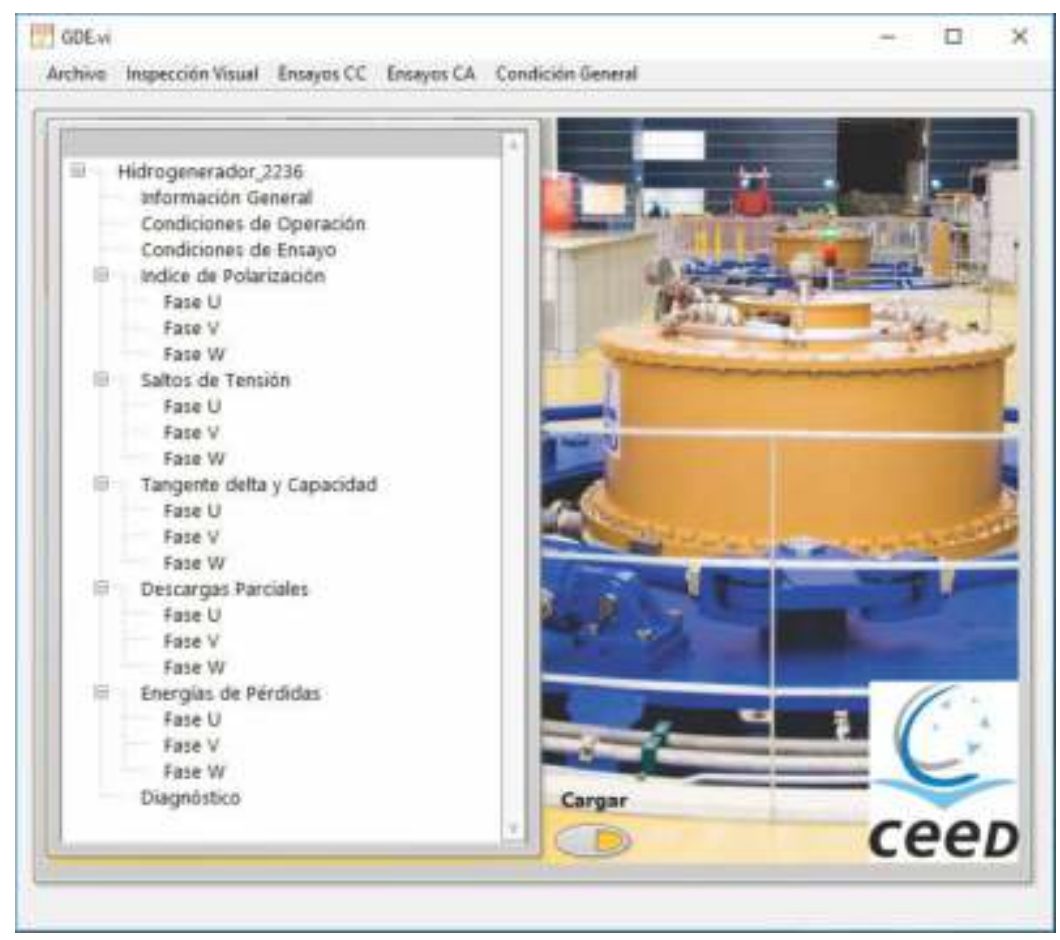

Fig. 2. Ventana Principal del GE.

En la Fig. 3 se presenta un esquema mediante un diagrama de flujo para el procesamiento de datos a partir de los resultados derivados del ensayo de Índice de Polarización (IP) [9].

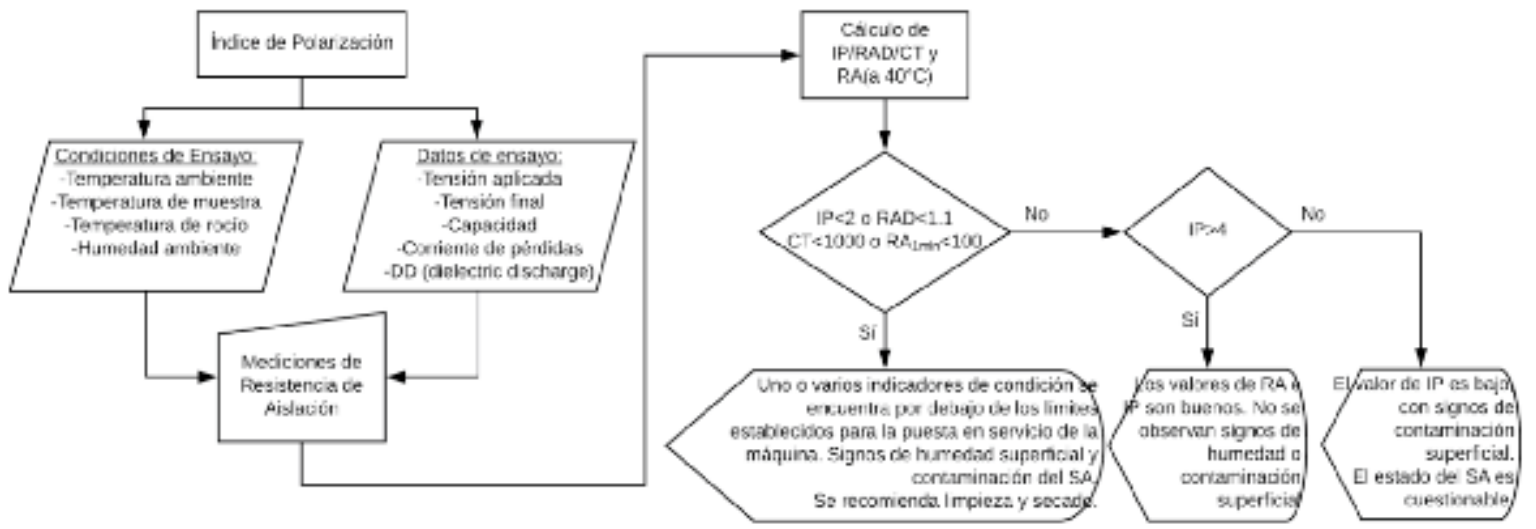

Fig. 3. Diagrama de Flujo para procesamiento de información de IP

Una vez codificado el diagrama de flujo en el entorno de desarrollo NI LabView, en la Figura 4 se muestra el panel frontal del gestor para visualizar los ensayos de Índice de Polarización. 


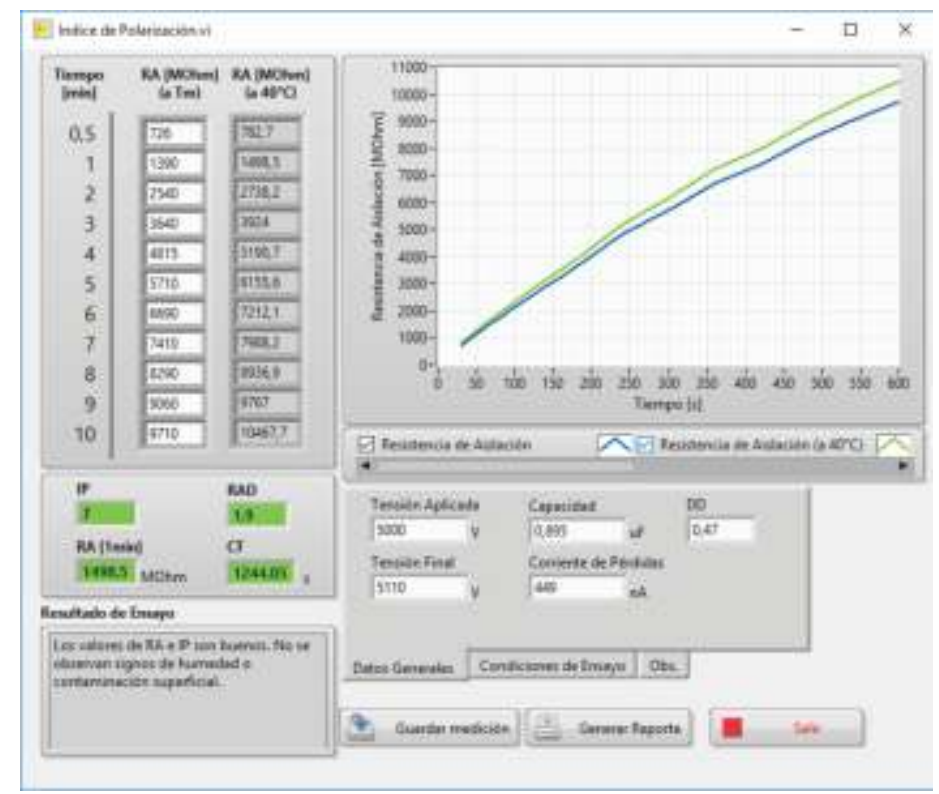

Fig. 4. Panel frontal ensayo IP

En los ensayos de saltos de tensión (ST) se evalúa la respuesta del sistema de aislación del bobinado ante cambios bruscos de tensión. La respuesta transitoria obtenida es un indicador del grado de contaminación del bobinado. La aplicación de este ensayo se hace bajo los criterios propuestos por la IRAM 2325, y el diagrama de flujo se observa en la Figura 5.

Una vez codificado el diagrama en el entorno de desarrollo LabView, en la Figura 6 se muestra el panel frontal del gestor para visualizar los ensayos de ST. El panel central permite visualizar gráficamente cada una de las mediciones para cada ensayo, así como, la posibilidad de almacenar datos y la generación automática de un reporte para su análisis.

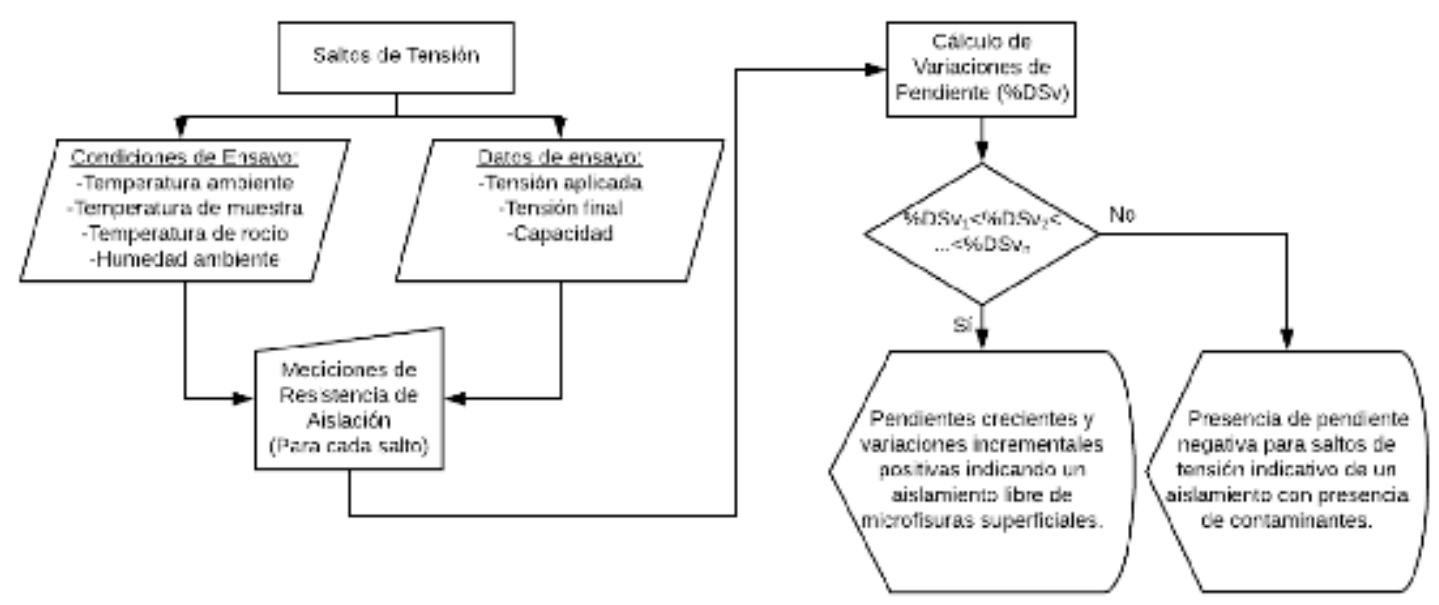

Fig. 5. Diagrama de Flujo ST 


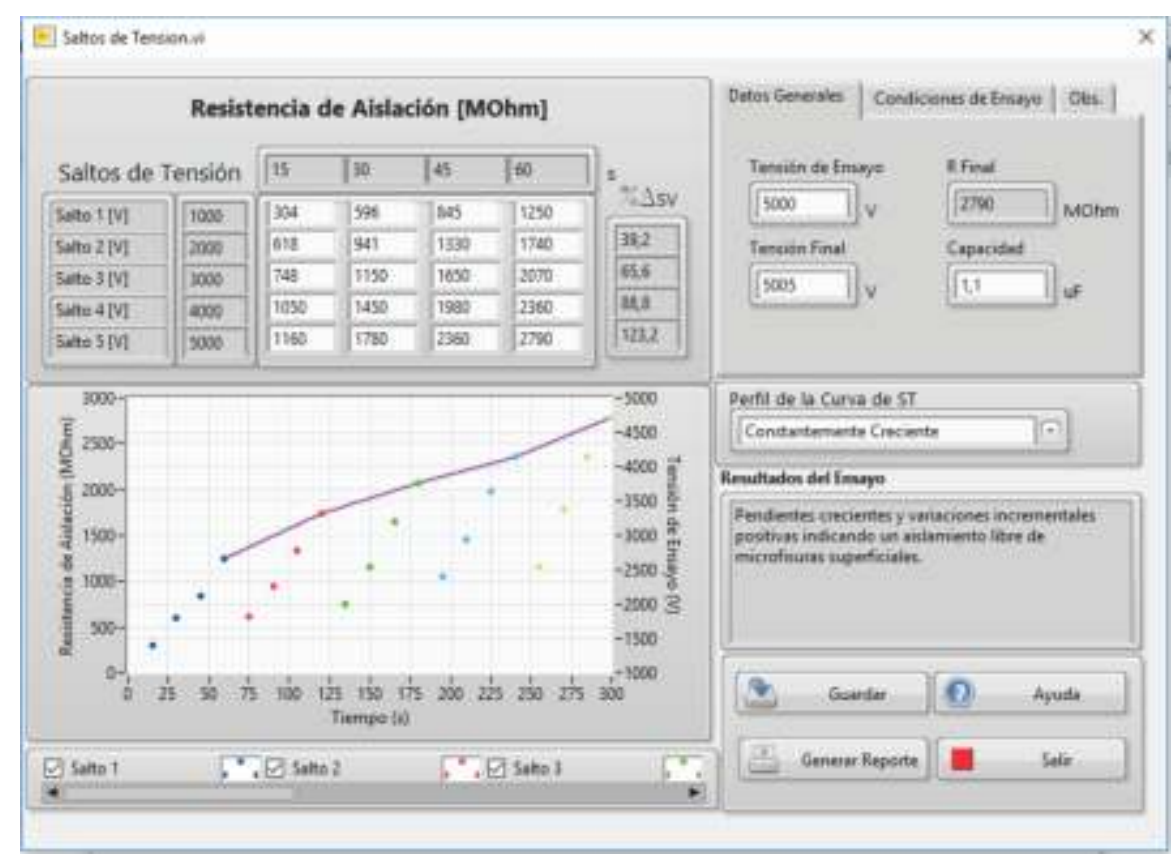

Fig. 6. Panel Frontal de Ensayo ST

Los ensayos de $\tan \delta$ y capacidad permiten evaluar el grado de contaminación volumétrica y la presencia de descargas parciales internas del aislante. El GE comparará los valores obtenidos en estos ensayos con los límites establecidos por la práctica [2], y normativas de referencia (VDE0530, IEEE Std. 286). De acuerdo a estos límites preestablecidos por normas, en la Figura 7 se muestra el diagrama de flujo elaborado.

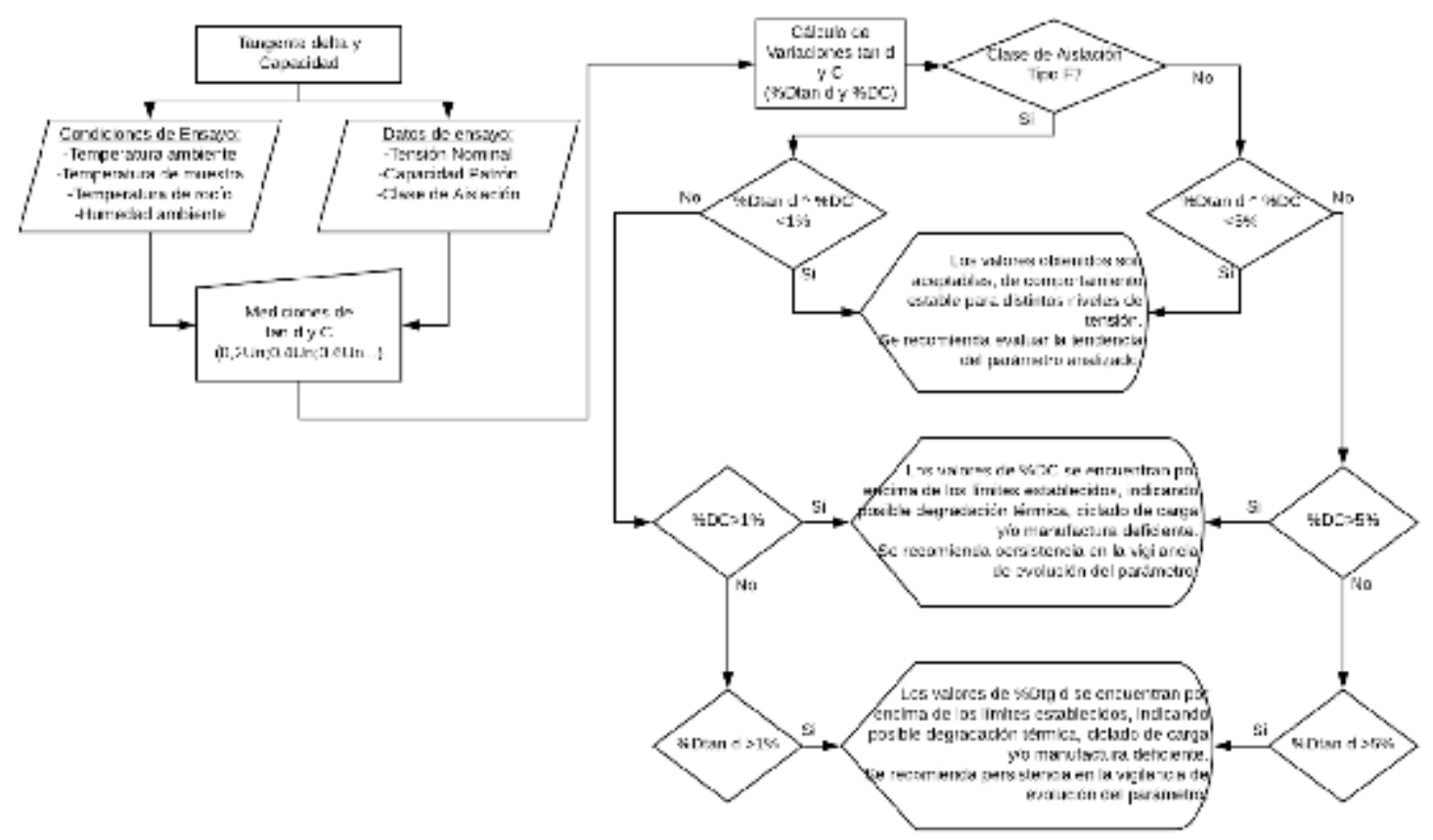

Fig. 7. Diagrama de Flujo Tan $\delta$ y $C$ 
En la Figura 7 se muestra el panel frontal del gestor para visualizar los ensayos de tan $\delta$ y capacidad. El panel central permite comparar los valores medidos con los límites de referencias (VDE, Marelli, EdF).

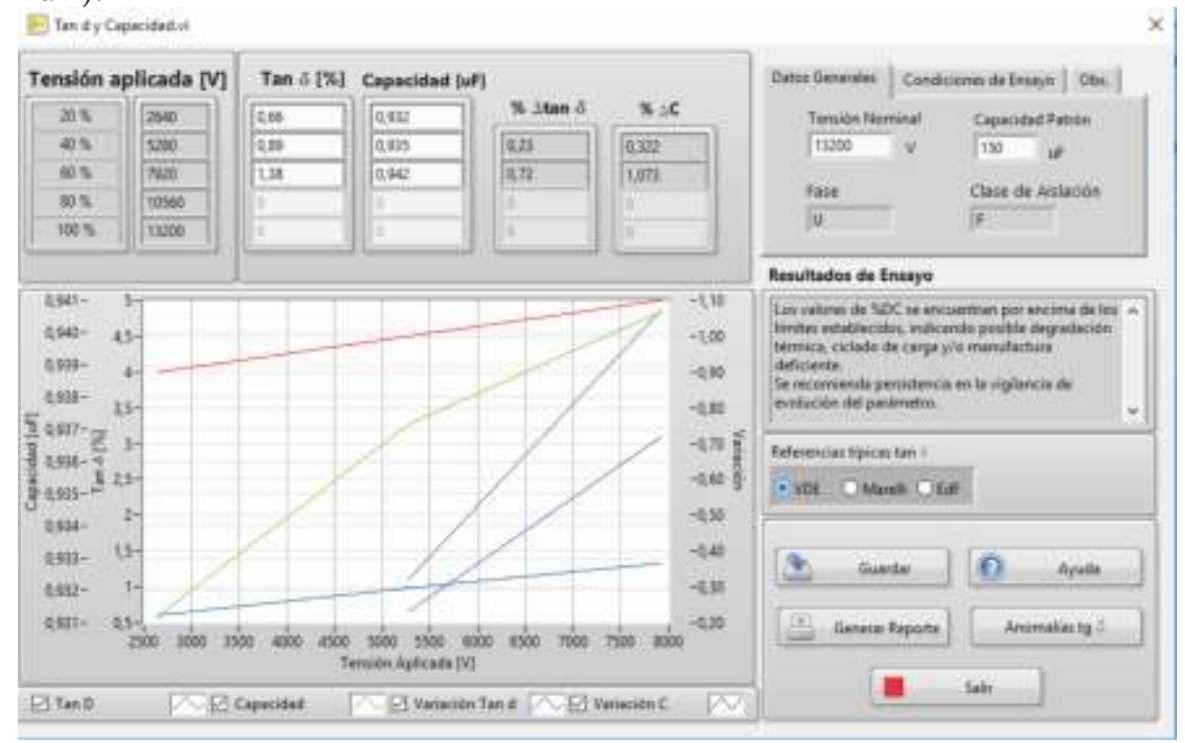

Fig. 8. Panel Frontal de Ensayo Tan $\delta$ y $C$

El ensayo permite evaluar la degradación del sistema aislante, daños en la película semiconductora y procesos de delaminación. Las consideraciones para evaluar los resultados del ensayo de DP se encuentran en los estándares IEEE Std. 1443, IEC 60034-27, recomendaciones de organismos como CIGRE, IEEE, CRIEPI y especialistas. En la Figura 9 se presenta el diagrama de flujo propuesto.

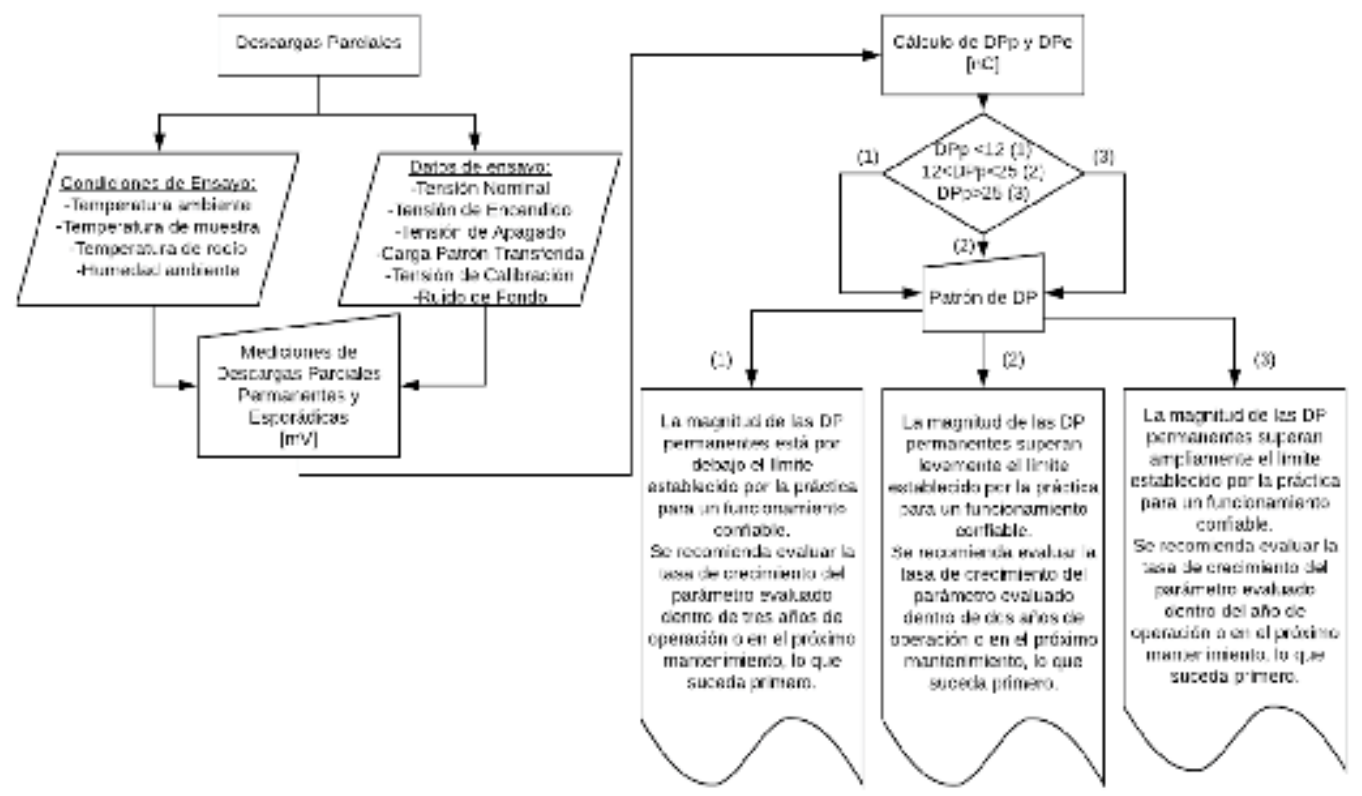

Fig. 9. Diagrama de Flujo DP

La magnitud de las descargas parciales permanentes puede derivar en tres tipos de diagnósticos dando como recomendación el tiempo de evaluación de la tasa de crecimiento del parámetro 
evaluado. Es de gran importancia evaluar la tendencia en el tiempo respecto de mediciones anteriores. Adicionalmente, se evalúa la clase o patrón de la descarga que son indicativos de la severidad que representa la descarga al sistema de aislación. En la Figura 10 se muestra el panel para visualizar los ensayos de DP.

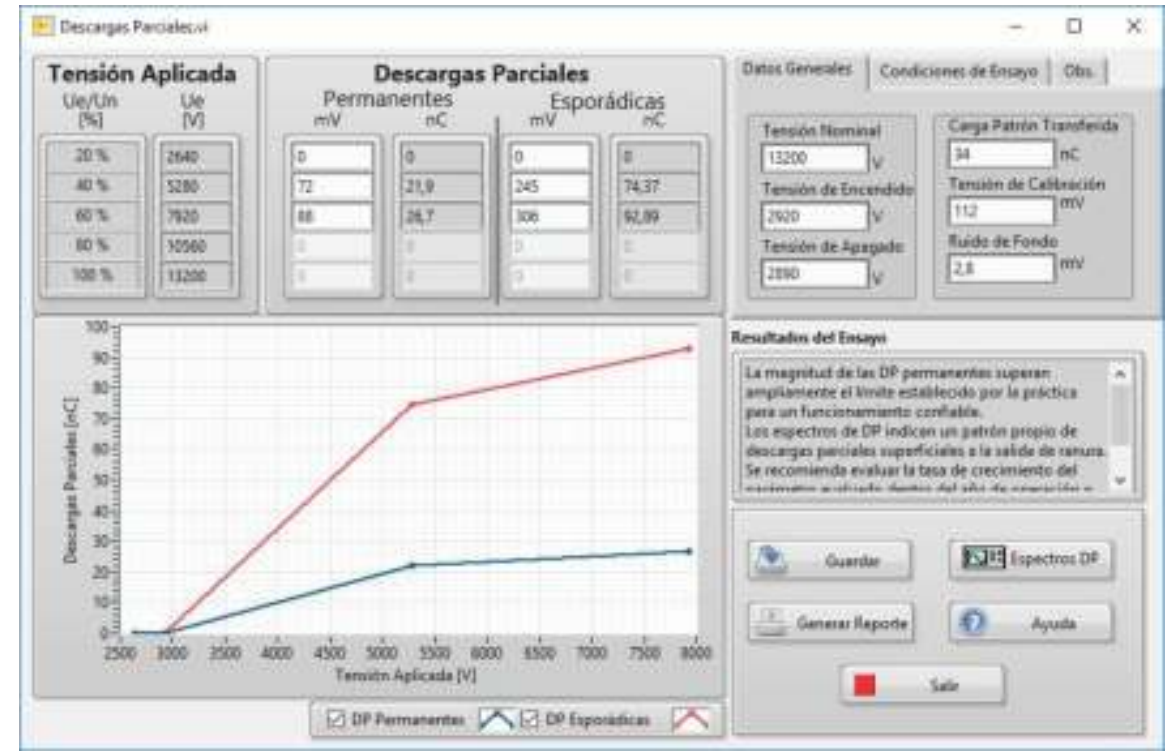

Fig. 10. Panel Frontal de Ensayo DP

El ensayo de Energía Integrada de las Descargas consiste en la medición de la suma de las energías en $\mu \mathrm{J} / \mathrm{pF}$ de todas las descargas parciales producidas durante un ciclo. Esta magnitud es representativa de la actividad de descargas en la aislación y un incremento en su valor podría estar indicando un la existencia o empeoramiento de un proceso de degradación. La metodología empleada es el denominado Método del lazo, de acuerdo con lo planteado en la ASTM D 3382. En la Figura 11 se muestra el panel para visualizar los ensayos de Energía Integrada de las DP.

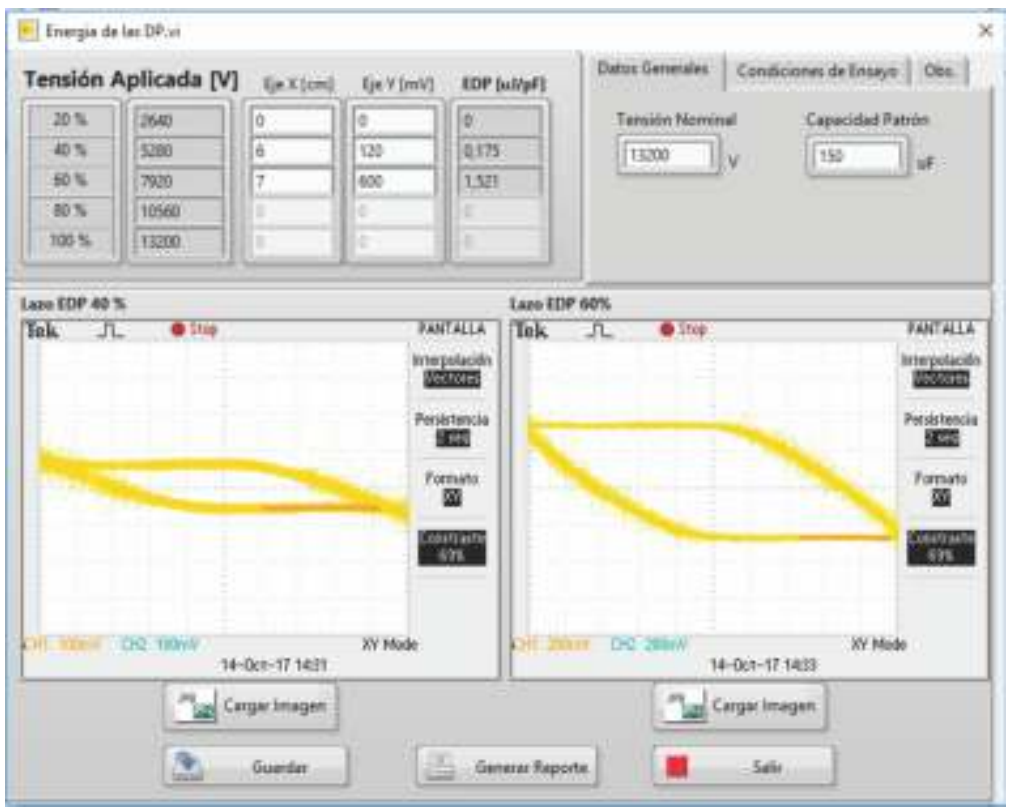

Fig. 11. Panel Frontal de Energía de las DPs 
A partir de la valoración de los IC (entre 1 y 5) y de la ecuación (1), el gestor de ensayos realiza la compilación de los mismos y visualiza el estado general de la máquina. Esta herramienta indica la condición general de la máquina permitiendo establecer un orden de prioridad de intervención.

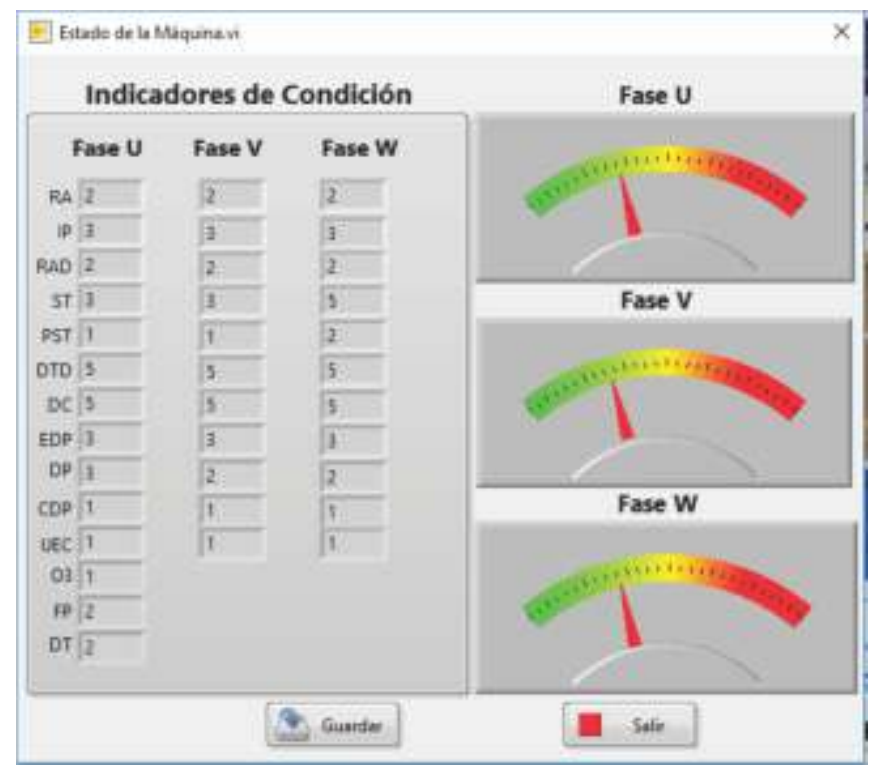

Fig. 12. Estado de la Máquina según los IC

\section{Conclusiones}

En este trabajo se presentó un modelo determinístico basado en indicadores de condición para la evaluación integral del estado de sistemas de aislación estatórico en hidrogeneradores. Con el fin de establecer un Orden de Prioridad (OP) de intervención para realizar operaciones de mantenimiento, 14 indicadores de condición fueron obtenidos de ensayos dieléctricos off-line, inspecciones y verificaciones poco invasivas. Los resultados obtenidos demuestran que existen cuatro unidades que manifiestan la presencia de DP en ranura por pérdida de película semiconductora, lo cual se confirma indirectamente por la presencia de elevados niveles de concentración de ozono en el recinto del generador. Si bien este mecanismo de degradación es de evolución lenta, el mismo es de difícil mitigación y es menester realizar una evaluación detallada del grado de avance de dicho mecanismo de degradación. El quinto orden de prioridad se otorga a la unidad \#7 la cual ha presentado indicadores de contaminación superficial y degradación térmica. Este último mecanismo de degradación podría estar relacionado con un sistema de refrigeración deficiente y/o por el ciclado térmico de la unidad. Las últimas tres unidades presentan indicadores de condición similares, con similitud en el OP. Particularmente en las unidades \#3 y \#8 el OP resultante es el mismo, debido a que los mecanismos de degradación en ambas máquinas han evolucionado de manera similar. A fin de poder discernir un orden de prioridad entre ambas máquinas, se deberá incorporar ensayos y verificaciones adicionales como son: análisis del historial de mantenimiento, monitoreo on-line de DP, vibraciones en núcleo estatórico y cabezas de bobina, mapeo de cuñas, DC Hi-Pot, inspección ultrasónica para detección de corona, entre otros.

Se desarrolló un Gestor de Ensayos Dieléctricos para Máquinas Eléctricas Rotativas (GE) que permitió visualizar de forma automática el diagnostico final del sistema de aislación de la máquina. 
Cabe mencionar que la metodología propuesta considera solo los ensayos dieléctricos off-line, con lo cual, se pretende, en trabajos futuros, incluir información complementaria proveniente de los ensayos del tipo on-line (vibraciones, descargas parciales on-line, medición de entrehierro, etc.), como así también, la aplicación a máquinas eléctricas estáticas.

\section{Agradecimientos}

Los autores agradecen al CEED (Centro de Estudios de Energía para el Desarrollo) y a la Facultad de Ingeniería de la UNaM.

\section{Referencias}

[1] "Survey of Hydrogenerator Failures" Working Group A1.10. CIGRÉ, 2009.

[2] Greg C. Stone; Ian Culbert; Edward A. Boulter; Hussein Dhirani. Electrical Insulation for Rotating Machines: Design, Evaluation, Aging, Testing, and Repair, 1, Wiley-IEEE Press, 2014, pp. 672.

[3] EPRI Hydro Life Extension Modernization Guide. Volume 3: Electromechanical Equipment. December 2001.

[4] ASTM D3382-07 Standard Test Method for Measurement of Energy and Integrated Charge Transfer Due to Partial Discharges (Corona) Using Bridge Techniques.

[5] IE (Cigre, 2009) C 60034-27:2006 Off-line partial discharge measurement on the stator winding insulation of rotating electrical machines.

[6] C. Millet, D. N. Nguyen, L. Lepine, M. Belec, D. Lessard-Deziel and C. Guddemi, "Case study - high ozone concentration in hydro generators," 2009 IEEE Electrical Insulation Conference, Montreal, QC, 2009, pp. 178182.

[7] G. C. Stone, C. V. Maughan, D. Nelson and R. P. Schultz, "Impact of Slot Discharges and Vibration Sparking on Stator Winding Life in Large Generators," in IEEE Electrical Insulation Magazine, vol. 24, no. 5, pp. 14-21, September-October 2008.

[8] C. Hudon, M. Belec, C. Guddemi, S. Lamothe, S. Nguyen and D. N. Nguyen, "Restoration of ground-wall armor of stator bars exposed to slot discharges," Conference Record of the the 2002 IEEE International Symposium on Electrical Insulation (Cat. No.02CH37316), 2002, pp. 497-501.

[8] IEEE. (2013). Recommended Practice for Testing Insulation Resistance of Rotating Machinery. IEEE Std 432013. 